Blood. 2011;117(17):4632-4641.

6. Shaheen M, Broxmeyer HE. Hematopoietic cytokines and growth factors. In: Broxmeyer HE, eds. Cord Blood Biology, Transplantation, Banking, and Regulation. Bethesda, Maryland, USA:AABB Press; 2011:35-74

7. Shaheen M, Broxmeyer HE. Principles of cytokine signaling. In: Hoffman R, Benz EJ Jr, Silberstein LE, Heslop H, Weitz JI, Anastasi J, eds. Hema tology: Basic Principles and Practice. 6th ed. Philadelphia, Pennsylania, USA: Elsevier Saunders; 2013:136-146.

8. Broxmeyer HE, et al. Dipeptidylpeptidase 4 negatively regulates colony-stimulating factor activity and stress hematopoiesis. Nat Med. 2012; 18(12):1786-1796

9. Ou X, O'Leary HA, Broxmeyer HE. Implications of DPP4 modification of proteins that regulate stem/progenitor and more mature cell types. Blood. 2013;122(2):161-169.

10. Christopherson KW 2nd, Hangoc G, Mantel CR,
Broxmeyer HE. Modulation of hematopoietic stem cell homing and engraftment by CD26. Science. 2004;305(5686):1000-1003.

11. Csaszar E, et al. Rapid expansion of human hematopoietic stem cells by automated control of inhibitory feedback signaling. Cell Stem Cell. 2012; 10(2):218-229

12. Guo Y, Mantel C, Hromas RA, Broxmeyer HE. Oct 4 is critical for survival/antiapoptosis of murine embryonic stem cells subjected to stress, effects associated with Stat3/Survivin. Stem Cells. 2008; 26(1):30-34.

13. Sack MN, Finkel T. Mitochondrial metabolism, sirtuins, and aging. Cold Spring Harb Perspect Biol. 2012;4(12):a013102.

14. Han M-K, Song EK, Guo Y, Ou X, Mantel C, Broxmeyer HE. SIRT1 regulates apoptosis and Nanog expression in mouse embryonic stem cells by controlling p53 subcellular localization. Cell Stem Cell. 2008;2(3):241-251

15. Zhang ZN, Chung SK, Xu Z, Xu Y. Oct4 maintains the pluripotency of human embryonic stem cells by inactivating p53 through Sirt1-mediated deacetylation. Stem Cells. 2014;32(1):157-165.

16. Ou X, Lee MR, Huang X, Messina-Graham S, Broxmeyer HE. SIRT1 positively regulates autophagy and mitochondria function in embryonic stem cells under oxidative stress. Stem Cells. 2014; 32(5):1183-1194.

17. Ou X, et al. SIRT1 deficiency compromises mouse embryonic stem cell hematopoietic differentiation, and embryonic and adult hematopoiesis in the mouse. Blood. 2011;117(2):440-450.

18. Broxmeyer HE, et al. A role for DEK in stem/progenitor cell biology. Stem Cells. 2013;31(8):1447-1453.

19. Li W, Li K, Wei W, Ding S. Chemical approaches to stem cell biology and therapeutics. Cell Stem Cell. 2013;13(3):270-283

20. Lee MR, et al. Epigenetic regulation of Nanog by miR-302 cluster-MBD2 completes induced pluripotent stem cell reprogramming. Stem Cells. 2013;31(4):666-681.

\title{
Unraveling the relationship between microbial translocation and systemic immune activation in HIV infection
}

\author{
Liang Shan ${ }^{1}$ and Robert F. Siliciano ${ }^{2,3}$
}

${ }^{1}$ Department of Immunobiology, Yale University, New Haven, Connecticut, USA. ${ }^{2}$ Department of Medicine, Johns Hopkins University School of Medicine, Baltimore, Maryland, USA. ${ }^{3}$ Howard Hughes Medical Institute, Baltimore, Maryland, USA.

Chronic immune activation is a key factor in HIV-1 disease progression. The translocation of microbial products from the intestinal lumen into the systemic circulation occurs during HIV-1 infection and is associated closely with immune activation; however, it has not been determined conclusively whether microbial translocation drives immune activation or occurs as a consequence of HIV-1 infection. In an important study in this issue of the $J C I$, Kristoff and colleagues describe the role of microbial translocation in producing immune activation in an animal model of HIV-1 infection, SIV infection of pigtailed macaques. Blocking translocation of intestinal bacterial LPS into the circulation dramatically reduced $T$ cell activation and proliferation, production of proinflammatory cytokines, and plasma SIV RNA levels. This study directly demonstrates that microbial translocation promotes the systemic immune activation associated with HIV-1/SIV infection.

\section{Immune activation and disease progression in HIV-1 infection}

In humans, the gut-associated lymphoid tissue (GALT) contains a large number of activated $\mathrm{CD}^{+}{ }^{+} \mathrm{CR} 5^{+} \mathrm{T}$ cells, thus serving as a major site of viral replication and $\mathrm{CD}^{+} \mathrm{T}$ cell depletion early in the course of HIV-1 infection $(1,2)$. In contrast to the rapid depletion of intestinal $\mathrm{CD} 4^{+} \mathrm{T}$ cells, the decline of $\mathrm{CD}^{+} \mathrm{T}$ cells in peripheral

Conflict of interest: The authors have declared that no conflict of interest exists.

Citation for this article: $J$ Clin Invest. 2014;

124(6):2368-2371. doi:10.1172/JCI75799. blood is much slower and less extensive during acute HIV-1 infection, suggesting that the majority of peripheral $\mathrm{CD} 4^{+} \mathrm{T}$ cells are nonpermissive at this stage $(2,3)$. During the chronic phase of infection, systemic immune activation is very strongly correlated with disease progression (4). Activation of $\mathrm{CD}^{+} \mathrm{T}$ cells increases coreceptor expression and renders these cells more susceptible to HIV-1 infection, and these cycles of activation and infection may drive progression of disease to AIDS (5). In contrast, SIV infection in natural host species very rarely progresses to AIDS and does not result in increased levels of immune activation, despite high levels of virus replication $(6,7)$. Infection and depletion of $\mathrm{CD}^{+} \mathrm{T}$ cells in GALT can be observed in both SIV and HIV-1 infection; however, natural host species infected with SIV do not often develop systemic immune activation, despite loss of GALT CD4 ${ }^{+} \mathrm{T}$ cells $(6,7)$, suggesting that factors other than direct infection and mucosal $\mathrm{CD} 4^{+} \mathrm{T}$ cell loss are essential for the increased degree of immune activation seen in HIV-1 infection.

\section{Microbial translocation in HIV-1 infection}

Translocation of microbial products from the lumen of the gastrointestinal tract into the circulation often occurs in HIV-1infected individuals and is closely associated with systemic immune activation. Many studies have shown that HIV-1infected individuals have elevated plasma levels of LPS and soluble CD14, which is indicative of LPS-induced monocyte and macrophage activation (8). Plasma levels of other microbial products, such as bacterial DNA and flagellin, are also increased in HIV-1-infected individuals compared with healthy controls $(8,9)$. Furthermore, there is a negative correlation between plasma 
A
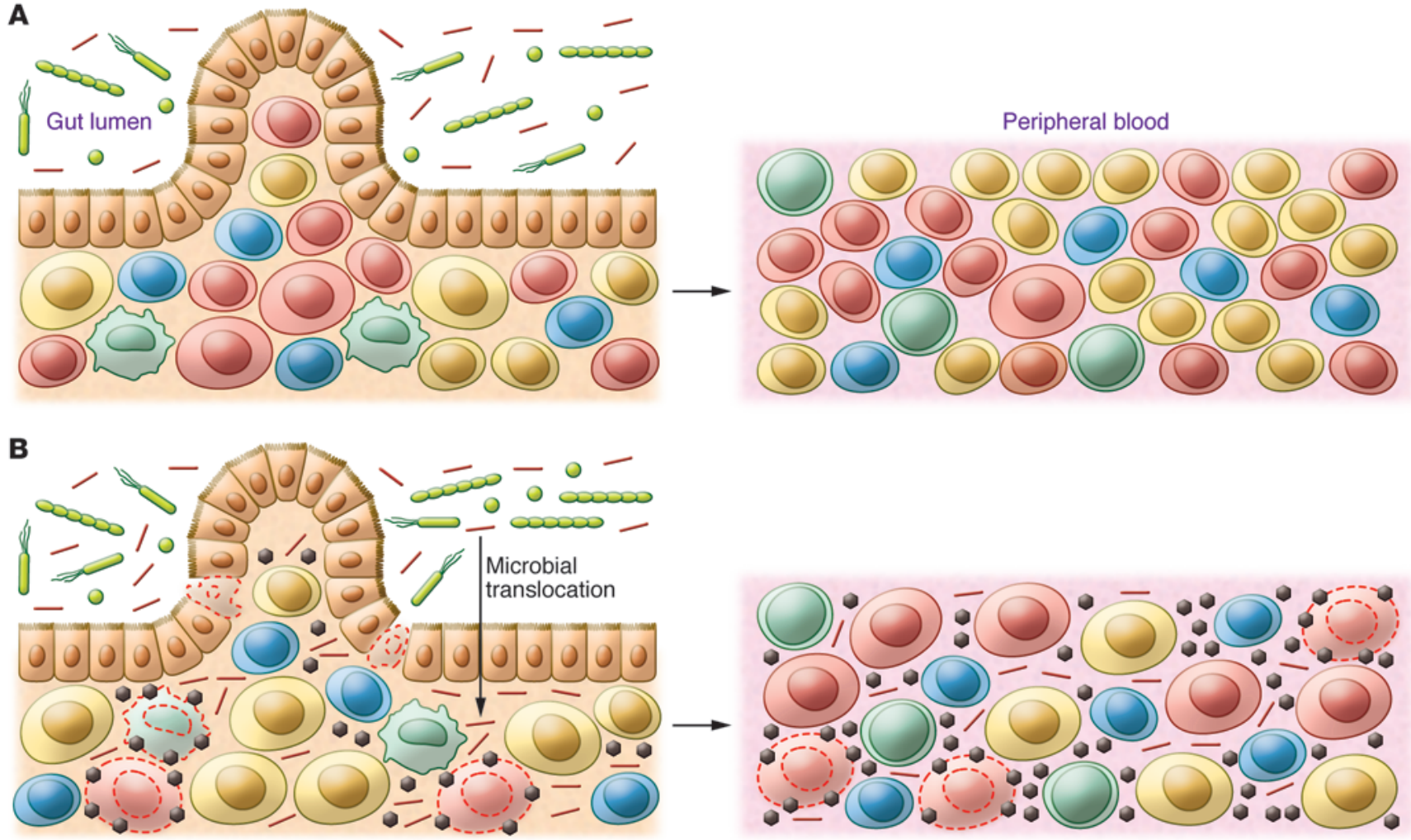

C
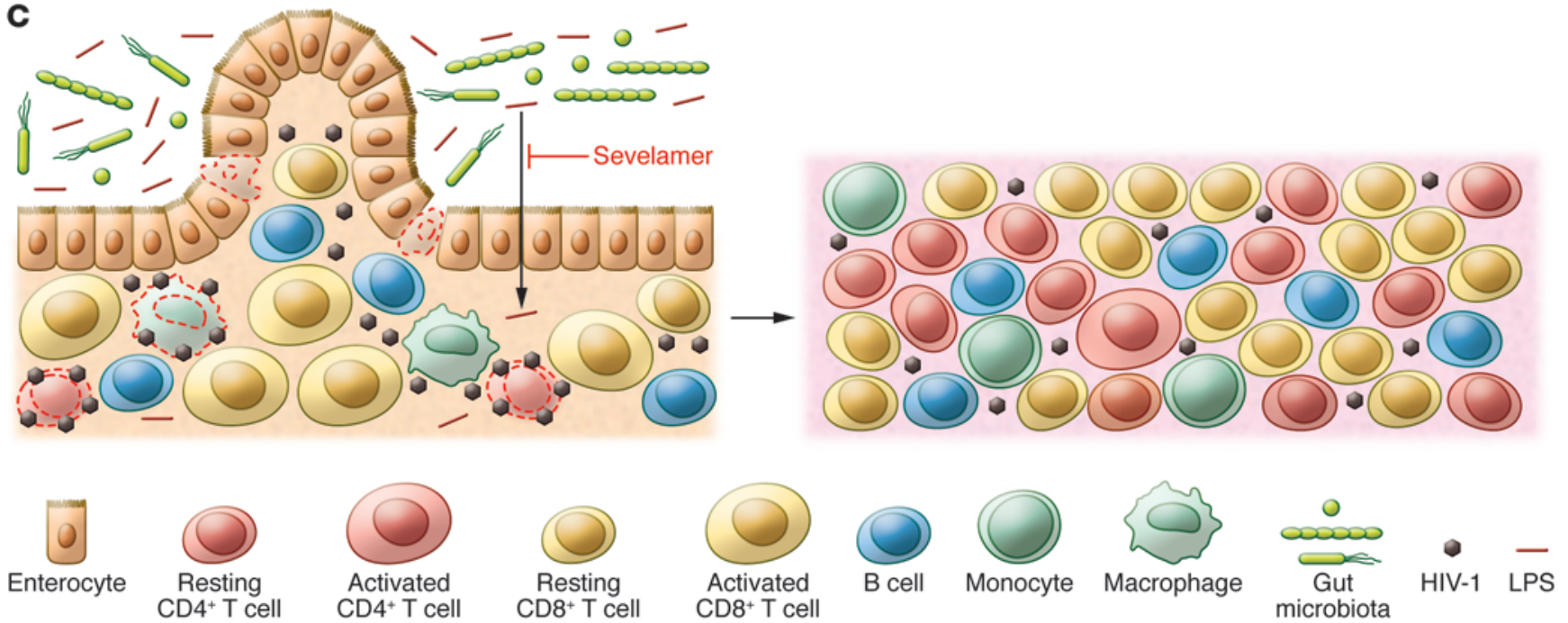

Figure 1

Microbial translocation and immune activation during HIV-1 infection. (A) The intact intestinal epithelium in uninfected individuals. Commensal bacteria and released microbial products, including LPS, are restricted to the gut lumen by a continuous lining of enterocytes. (B) Translocation of commensal bacteria and bacterial products from the gut lumen into the intestinal wall and peripheral blood during HIV-1 infection. HIV-1 infection induces death of intestinal epithelial cells and depletes intestinal CD4+ T cells, particularly Th17 cells. Loss of intestinal epithelial cells allows commensal bacteria to enter intestinal wall. Loss of Th17 cells allows bacterial overgrowth. Translocation of microbial products into peripheral blood causes systemic immune activation. Activated peripheral CD4+ T cells are highly susceptible to HIV-1 infection. (C) The drug sevelamer blocks translocation of bacterial LPS into the systemic circulation, preventing immune activation. Sevelamer binds and sequesters bacterial LPS in intestinal lumen. Blocking bacterial LPS translocation of LPS into the circulation prevents systemic immune activation, leaving the majority of peripheral CD4+ $\mathrm{T}$ cells quiescent and unsusceptible to HIV-1 infection. 
levels of LPS and CD4 ${ }^{+} \mathrm{T}$ cell counts (10). The cause of microbial translocation is multifactorial. HIV-1 replicates vigorously in GALT and causes damage to intestinal barrier through several mechanisms. First, HIV-1 infection leads to the production of proinflammatory cytokines, which induce apoptosis of enterocytes and disruption of tight junctions, the epithelial barrier against microbial translocation (11). A second mechanism involves intestinal Th17 cells, which produce cytokines, including IL-17 and IL-22, to recruit and activate neutrophils as well as promote the release of antimicrobial peptides (12). Th17 cells are preferentially depleted by direct infection, likely due to the high expression of the CCR5 coreceptor on these cells (13). As a consequence of viral infection, damage to intestinal epithelial barrier and decreased control of intestinal bacteria result in the translocation of commensal microbial products, including LPS, into circulation. In principle, these products can induce production of proinflammatory cytokines and promote peripheral $\mathrm{T}$ cell activation and cycling (Figure 1). Microbial translocation occurs during HIV-1 and pathogenic SIV infection but not during SIV infection of natural host species (5), suggesting that microbial translocation may play a role in viral infection and disease progression. Interestingly, intravenous LPS administration in natural host species infected with SIV does induce systemic immune activation, increase SIV replication, and accelerate $\mathrm{CD}^{+} \mathrm{T}$ cell depletion (14). Nevertheless, it is unclear whether and to what degree microbial translocation drives immune activation and disease progression or if the breach of epithelial barrier function occurs concomitantly with immune activation as a consequence of viral infection.

\section{Microbial translocation is indispensable for HIV-1-associated systemic immune activation}

Experiments to directly evaluate the contribution of microbial translocation in the pathogenesis of HIV-1 infection require blocking the interaction between microbial products and host immune cells without directly affecting viral replication. In this issue, Kristoff and colleagues used SIV-infected pigtailed macaques (PTMs) and the drug sevelamer as a model to investigate the role of microbial translocation in immune activation and viral replication in disease progression (15). Seve- lamer is a cationic polyallylamine polymer that shows a high capacity for binding anions such as phosphate and can also bind and sequester LPS in the gut lumen. Furthermore, sevelamer has been used for the treatment of hyperphosphatemia in patients with chronic kidney disease (16). As evidenced by plasma levels of LPS and soluble CD14, sevelamer effectively, if not completely, blocked translocation of LPS into circulation of SIV-infected PTMs. In sevelamer-treated animals, reduced levels of LPS were associated with decreased $\mathrm{T}$ cell activation and proliferation and decreased production of proinflammatory cytokines (15), suggesting that microbial translocation directly causes viral-associated systemic immune activation. Kristoff et al. further investigated whether blocking microbial translocation had an effect on viral replication and disease progression. While sevelamer treatment had only a limited effect on plasma SIV RNA levels during the first 2-3 weeks of infection, viral replication was significantly inhibited (by more than one log) in sevelamer-treated animals at time points beyond 3 weeks after infection. Reduced viral replication occurred concomitantly with reduction of peripheral $T$ cell activation (15), suggesting that blocking microbial translocation reduces systemic SIV replication through limiting $\mathrm{T}$ cell activation (Figure 1). Although this study by Kristoff et al. demonstrates that translocation of LPS is an important instigator of systemic immune activation, it remains to be determined whether and to what degree other microbial products, including peptidoglycan, lipoteichoic acid, and flagellin, also contribute to immune activation. Additionally, it is not clear whether inhibiting microbial translocation during the acute phase of SIV infection would change the clinical outcome of chronic infection; therefore, long-term follow-up after treatment seems warranted. Nonetheless, this study by Kristoff and colleagues provides direct evidence that microbial translocation promotes immune activation in SIV-infected PTMs and suggests that therapeutic interventions to decrease microbial translocation in HIV-1-infected individuals could provide benefit.

\section{Conclusions and future directions}

Current combinational antiretroviral therapy (cART) can suppress HIV-1 replication to undetectable levels; however, long-term cART fails to completely repair virus- induced gastrointestinal tract damage or restore intestinal $\mathrm{CD}^{+} \mathrm{T}$ cell populations $(17,18)$. Microbial translocation still occurs despite suppressed viral replication in cART-treated patients. Plasma LPS levels in HIV-1-infected individuals decline after initiation of cART but remain substantially higher than those seen in uninfected subjects (19). Plasma LPS and bacteria DNA levels in cART-treated patients are correlated inversely with $\mathrm{CD}^{+} \mathrm{T}$ cell reconstitution in the $\operatorname{GALT}(9,20)$, suggesting that therapeutic interventions to block microbial translocation in cART-treated patients may be important for complete reconstitution of gastrointestinal immunity. Kristoff and colleagues demonstrate that sevelamer can effectively block LPS translocation in SIV-infected PTMs and alleviate systemic immune activation during acute infection. It will be important to further investigate the effects of sevelamer on mucosal immune reconstitution in chronically infected PTMs. Because sevelamer is approved to treat hyperphosphatemia in patients with chronic kidney disease, it may be reasonable to assess whether sevelamer can attenuate immune activation and promote restoration of mucosal immunity in some cART-treated patients, especially immunological nonresponders, who maintain high levels of microbial translocation and immune activation and fail to restore $\mathrm{CD}^{+} \mathrm{T}$ cell population despite long-term $\operatorname{cART}(9,21)$.

\section{Acknowledgments}

This work was supported by NIH grant T32 AI07019 (to L. Shan), the Martin Delaney Collaboratory of AIDS Researchers for Eradication and the Delaney AIDS Research Enterprise (NIH grants AI096113 and AI096109 to R.F. Siliciano), the Foundation for AIDS Research grant 108165-50RGRL (to R.F. Siliciano), NIH grant 43222 (to R.F. Siliciano), and the Howard Hughes Medical Institute (to R.F. Siliciano).

Address correspondence to: Robert F. Siliciano, Johns Hopkins University School of Medicine, 733 North Broadway, BRB 879, Baltimore, Maryland 21205, USA. Phone: 410.955.2958; Fax: 410.955.0964; E-mail: rsiliciano@jhmi.edu.

1. Veazey RS, et al. Gastrointestinal tract as a major site of $\mathrm{CD}^{+} \mathrm{T}$ cell depletion and viral replication in SIV infection. Science. 1998;280(5362):427-431.

2. Smit-McBride Z, Mattapallil JJ, McChesney M, Ferrick D, Dandekar S. Gastrointestinal T lymphocytes retain high potential for cytokine responses but have severe CD4(+) T-cell depletion at all 
stages of simian immunodeficiency virus infection compared to peripheral lymphocytes.J Virol. 1998;72(8):6646-6656.

3. Mehandru S, et al. Primary HIV-1 infection is associated with preferential depletion of $\mathrm{CD}^{+} \mathrm{T}$ lymphocytes from effector sites in the gastrointestinal tract. J Exp Med. 2004; 200(6):761-770.

4. Giorgi JV, et al. Shorter survival in advanced human immunodeficiency virus type 1 infection is more closely associated with $\mathrm{T}$ lymphocyte activation than with plasma virus burden or virus chemokine coreceptor usage. J Infect Dis. 1999;179(4):859-870.

5. Sodora DL, Silvestri G. Immune activation and AIDS pathogenesis. AIDS. 2008;22(4):439-446.

6. Gordon SN, et al. 2007. Severe depletion of mucosal $\mathrm{CD}^{+} \mathrm{T}$ cells in AIDS-free simian immunodeficiency virus-infected sooty mangabeys. J Immunol. 2007;179(5):3026-3034.

7. Silvestri G, et al. Nonpathogenic SIV infection of sooty mangabeys is characterized by limited bystander immunopathology despite chronic highlevel viremia. Immunity. 2003;18(3):441-452.

8. Sandler NG, Douek DC. Microbial translocation in HIV infection: causes, consequences and treatment opportunities. Nat Rev Microbiol. 2012;10(9):655-666.

9. Jiang W, et al. Plasma levels of bacterial DNA correlate with immune activation and the magnitude of immune restoration in persons with antiretroviral-treated HIV infection. J Infect Dis. 2009;199(8):1177-1185

10. Nowroozalizadeh S, et al. Microbial translocation correlates with the severity of both HIV-1 and HIV-2 infections. J Infect Dis. 2010;201(8):1150-1154.

11. Epple HJ, et al. Acute HIV infection induces mucosal infiltration with $\mathrm{CD}^{+}$and $\mathrm{CD}^{+}{ }^{+} \mathrm{T}$ cells, epithelial apoptosis, and a mucosal barrier defect. Gastroenterology. 2010;139(4):1289-1300.

12. Korn T, Bettelli E, Oukka M, Kuchroo VK. IL-17 and Th17 cells. Annu Rev Immunol. 2009;27:485-517.

13. Brenchley JM, et al. Differential Th17 CD4 T-cell depletion in pathogenic and nonpathogenic lentiviral infections. Blood. 2008;112(7):2826-2835.

14. Pandrea I, et al. Cutting edge: experimentally induced immune activation in natural hosts of simian immunodeficiency virus induces significant increases in viral replication and $\mathrm{CD}^{+} \mathrm{T}$ cell depletion. J Immunol. 2008;181(10):6687-6691.

15. Kristoff J, et al. Early microbial translocation block- ade reduces SIV-mediated inflammation and viral replication. J Clin Invest. 2014;124(6):2802-2806.

16. Sun PP, Perianayagam MC, Jaber BL. Endotoxin-binding affinity of sevelamer: a potential novel anti-inflammatory mechanism. Kidney Int Suppl. 2009;76(114):S20-S25.

17. Estes J, et al. Collagen deposition limits immune reconstitution in the gut. $J$ Infect Dis. 2008; 198(4):456-464.

18. Mehandru S, et al. Lack of mucosal immune reconstitution during prolonged treatment of acute and early HIV-1 infection. PLoS Med. 2006;3(12):e484.

19. Brenchley JM, et al. Microbial translocation is a cause of systemic immune activation in chronic HIV infection. Nat Med. 2006;12(12):1365-1371.

20. Baroncelli S, et al. Microbial translocation is associated with residual viral replication in HAARTtreated $\mathrm{HIV}^{+}$subjects with $<50$ copies/ml HIV-1 RNA.J Clin Virol. 2009;46(4):367-370.

21. Marchetti G, et al. Microbial translocation is associated with sustained failure in $\mathrm{CD}^{+} \mathrm{T}$-cell reconstitution in HIV-infected patients on longterm highly active antiretroviral therapy. AIDS. 2008;22(15):2035-2038.

\title{
Targeting secondary immune responses to cetuximab: CD137 and the outside story
}

\author{
Julie E. Bauman ${ }^{1}$ and Jennifer R. Grandis²
}

${ }^{1}$ Department of Internal Medicine (Hematology/Oncology) and 2Department of Otolaryngology, University of Pittsburgh, Pittsburgh, Pennsylvania, USA.

\begin{abstract}
Cetuximab is a murine-human chimeric IgG1 $\mathrm{mAb}$ directed against the EGFR that is approved for use in patients with colorectal and head and neck carcinomas. While some patients benefit greatly from cetuximab, many do not; therefore, strategies to increase the efficacy of this drug are of great clinical interest. In this issue of the JCI, Kohrt and colleagues report a strategy for enhancing the secondary immune response to cetuximab that involves sequential targeting with an agonist $\mathrm{mAb}$ against CD137 expressed on NK and T cells.
\end{abstract}

\section{The inside story on cetuximab}

EGFR, also known as HER1, is a member of the erythroblastic leukemia viral oncogene homolog (ERBB) family of transmembrane receptor tyrosine kinases (RTK). Deregulation of EGFR signaling initiates aberrant growth, proliferation, and angiogenesis. The clinical development of mAbs targeting the extracellular ligand-binding domain and small molecules targeting the intracellular ATPase domain of EGFR was originally compelled by the association of EGFR overexpression with poor prognosis in multiple epithelial malignancies. Despite overexpression of EGFR in the vast major-

Conflict of interest: Jennifer Grandis receives laboratory research funding from Novartis.

Citation for this article: J Clin Invest. 2014; 124(6):2371-2375. doi:10.1172/JCI76264. ity of human aerodigestive cancers, cetuximab has only gained FDA approval for use in patients with head and neck squamous cell carcinoma (HNSCC) and WT KRAS colorectal cancer (CRC), with monotherapeutic activity observed in just $10 \%$ to $20 \%$ of patients. Clinical selection for cetuximab is confounded by the absence of a reliable biomarker to predict patient response. Mutations that result in constitutively activated EGFR are associated with patient responsiveness to small-molecule EGFR inhibitors in lung cancer; however, these mutations are rarely identified in HNSCC or CRC. Moreover, neither EGFR overexpression nor EGFR amplification predicts clinical benefit from cetuximab $(1,2)$. The discordance between EGFR target expression and the efficacy of target blockade by cetuximab has broadened investigation into the mechanisms of action and development of therapeutic resistance.

Initial strategies to enhance cetuximab activity have focused on the intracellular signaling hypothesis (Figure 1A), which suggests that de novo or compensatory activation of parallel RTKs (alternate HER family members, cMet, IGF1R, FGFR, VEGFR), downstream EGFR-signaling nodes (RAS, PI3K, STAT3, SRC), or cell cycle promoters (aurora kinase, CDK4/6) circumvents EGFR blockade in HNSCC preclinical models; therefore, coinhibition of these resistance nodes should enhance the activity of cetuximab (3). Cetuximab resistance has also been attributed to heterodimerization of EGFR with other HER proteins that potentially prevent recognition of EGFR by cetuximab as well as acquisition of gain-of-function PIK3CA mutations that activate signaling downstream of EGFR. In CRC patients, activating KRAS and BRAF mutations confer clinical cetuximab resistance. Progressive insight into the complexity and plasticity of the EGFR signaling network has propelled cetuximab-combination trials to evaluate the efficacy of cotargeting these purported resistance nodes (Table 1). 